\title{
The Present and Future Tread of MSM Intervention: A Qualitative Study in Hubei Province, China
}

Liu $X^{1}$, Wang $Y^{2}$, Zhou $W^{3}$, Mei $X^{4}$ and Tan $X^{5 *}$ ${ }^{1}$ School of Health Sciences, Wuhan University, China ${ }^{2}$ The Wuhan Centers for Disease Control \& Prevention, China

*Corresponding author: Xiaodong Tan; School of Health Sciences, Wuhan University, 115 St Donghu Road, Wuhan, China

Received: February 10, 2017; Accepted: March 17, 2017; Published: March 27, 2017

\begin{abstract}
Introduction: To discuss the present of the MSM Intervention in the Centers for Disease Control (CDCs) and Non-government Organizations (NGOs) and explore more effective intervention measures and methods among MSM.

Methods: An in-depth interview based on a semi-quantitative interview outline was conducted in the CDCs and NGOs. The key questions included (1) what is the reasons for inconsistent of knowledge and behaviors among MSM; (2) what is the primary problems in complementing the MSM intervention; (3) what are the advantages and disadvantages about MSM intervention; and (4) what are suggestions for MSM intervention in the future. Interview data were recorded and coded by two experienced researchers through QSR N Vivo 8.0 Software.
\end{abstract}

Results: A total of 10 participants were enrolled in the study; of those, 5 were from the CDCs, and 5 were from NGOs, with ages ranging from 25 to 52 . Four key themes on MSM intervention were identified with participants. These included (1) poor knowledge, small probability to infect HIVIAIDS and uncomfortable feelings contributed to the inconsistent of knowledge and behaviors; (2) the funds shortage, poor work efficiency and no unified evaluation criterion were the primary problems in MSM intervention; (3) the MSM intervention work in CDCs and NGOs should coordinate with each other; (4) the smart phone and new gay dating APP should be used in MSM intervention.

Conclusion: The existed intervention measures in CDCs and NGOs have not taken obvious effect on MSM, and it is necessary to explore new intervention approaches to change risk behavior of sexual. An approach of combining with the smart phone and APP should be used in MSM intervention in the future.

Keywords: MSM; CDCs; NGOs; AIDS; Intervention

\section{Introduction}

Men who have sex with men (MSM) are influenced by HIV/AIDS disproportionately in the global [1]. The rate of new HIV infection for MSM is 44 times than the general, and it still has a tendency to rise up [2]. Evidence-based HIV intervention measures have been developed and disseminated typically to meet the prevention and control needs among MSM [3]. In the developed countries, the Internet and new media had been used with HIV/AIDS prevention and care initiatives $[2,4,5]$ including sending health promotion messages, improving HIV/AIDS medication adherence, promoting test result notification and facilitating attendance at clinic appointments. The voice or text messaging functions had been utilized primarily for phone-based HIV intervention initially $[1,4,6,7]$ then more complex, tailored and interactive interventions were delivered more conveniently to target population through the software application and mobile web of smart-phones [2,5,7-14]. While in developing countries, publicity materials, peer education and condoms promotion were the mainly three intervention measures [15], with few researches conducted MSM intervention via the internet and new media $[16,17]$.

The purpose of MSM intervention is to protect MSM from infecting HIA/AIDS and promote health. Almost all of prevention measures including health promotion and health education, regular testing and sexual health care products promotion were used to MSM interventions [14,17]. However, these existed intervention or prevention measures have not taken obvious effect on MSM. In addition, only few researches paid an attention to the effect of the MSM intervention, and let alone the studies aimed on the intervention organizations. Even though several published articles on MSM had proved that their intervention measures were on effective3, there was still a growing trend of HIV epidemic among MSM, with all countries, states or provinces, and regions growing variously $[16,17]$.

While there was a body of literature on MSM intervention using quantitative approaches $[6,10,14,15,18,19]$ little had been done using qualitative approaches [20]. Existing researches most had been focused on the MSM $[17,20,21]$ rather than on the organizations of MSM intervention specifically. This is a qualitative study focused on the Centers for Disease Control (CDCs) and Non-government Organizations (NGOs) to discuss the MSM intervention in China. The aim of the study is to explore the participants' cognition MSM intervention and views of future prospect. And it is also our hope to explore more effective intervention methods and measures to increase AIDS/HIV knowledge and promote health among MSM. 


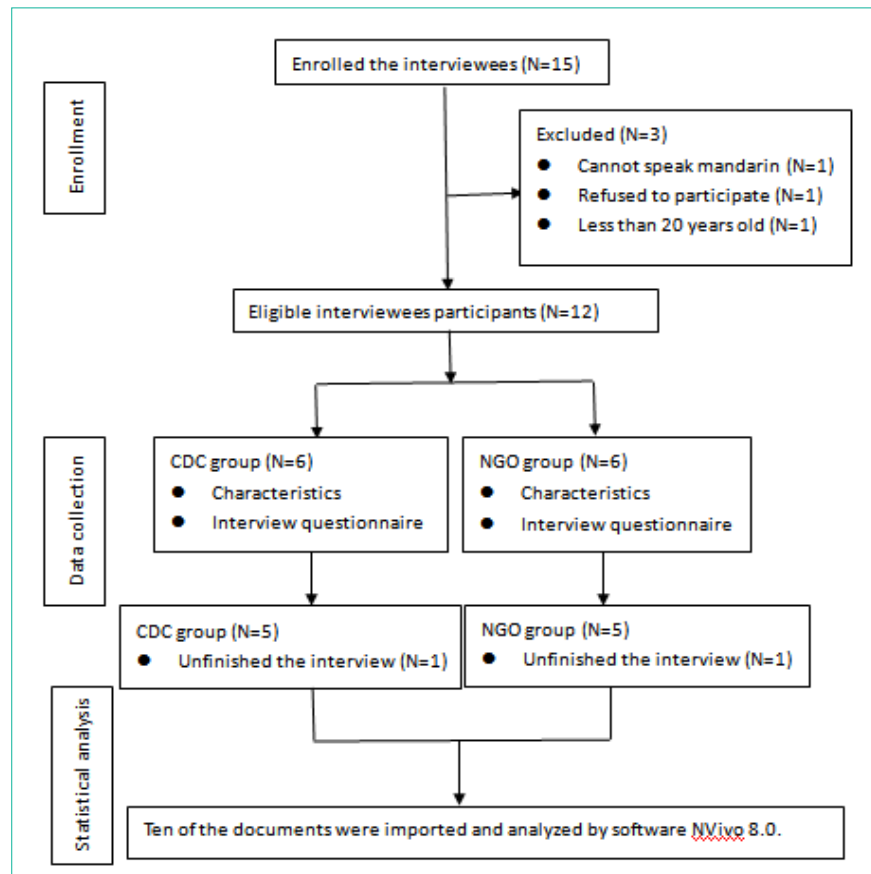

Figure 1: The procedure of study.

\section{Methods}

\section{Interview participants}

The participants who sought from the volunteers who worked for HIV/AIDS prevention and control in Wuhan city, Huangshi city, and Jingzhou city of Hubei province were recruited for an indepth interview. The candidates were invited by phone and told the intention of the research. There were 15 interviewees self-reported willing to participate the research, of which 7 were from Wuhan, 4 from Jingzhou and 4 from Huangshi. The eligible participants were those who worked more than 3 years in MSM intervention, with the age above 25 years old, and agreed to participate in the interview through signed consent. The interview participants who could not speak mandarin or who were unable to complete interview more than 20 minutes of an in-depth interview were excluded. Based on the above criterions, 10 interviewees were eligible to reach information saturation, with the CDC and NGO being 5 respectively. The whole study process was showed in (Figure 1).

\section{Ethical statements}

All subjects gave their informed consent for inclusion before they participated in the study. The study was conducted in accordance with the Declaration of Helsinki, and the protocol was approved by Medical Ethics Committees of School of Health Sciences, Wuhan University.

\section{Interview guide}

A semi-structured interview guide was developed based on the principles of the qualitative methodological approach, the objective of the study, and existing literatures on MSM intervention. According to the review and suggestion by two professors who had extensive experience in MSM intervention research and survey instrument development, revised the interview guide draft. Before the final interview, the revised guide was pilot tested with two volunteers--one from the CDC, and another from the NGO. Apart from the basic demographic questions, the final guide mainly explored the four aspects of questions, including: (1) what is the reasons for the inconsistent of knowledge and behaviors among MSM; (2) what is the primary problems in complementing the MSM intervention; (3) what are the advantages and disadvantages about MSM intervention in the CDCs and NGOs; and (4) what are suggestions for MSM intervention in the future.

\section{Data collection}

An in-depth interview based on a semi-quantitative interview outline was conducted by one of the researchers face-to-face, who were trained in interview and research protocols. The volunteers were recruited from the specialists in the fields of MSM intervention of the CDCs and NGOs in Hubei province. After being informed the study purpose and procedure of the interview, the eligible participants who expressed interest took part in the research voluntarily. Before the interview, one of the authors negotiated with the participants over interview time and place. Each of the participant in the CDCs were interviewed in an AIDS Voluntary Counseling and Testing room. While the participants in the NGOs were interviewed in one private office where the participant worked. Every interview lasted about 30 to 40 minutes. The whole interview was from October to December, 2014. The interview began with a self-introduction including name, gender, age, degree of education and working years, then asked a general questions --- what did you think about inconsistent of knowledge and behaviors among MSM; and then continued with specific questions --- what were problems you faced in the process of intervention and what were the differences of MSM intervention in the CDCs and NGOs, using their experiences for explanation; and eventually asked their advices for future MSM intervention. All the questions in the interview were open-ended and all the participants could speak freely. All interviews were recorded all over the process and transcribed by one of the researches into documents verbatim on the same day. Finally, 10 documents were produced for statistical analysis.

\section{Statistical analysis}

The software N Vivo 8.0 (QSR International Pty Ltd., Melbourne, Australia) was used to analyze the interview data, and the analysis process showed in (Figure 2). The detail steps were included: (1) Ten of transcribed documents were imported into software N Vivo 8.0 and repeatedly read by two authors who coded and analyzed thematically. (2) The unified coding rules were developed by the research team based on the content of the interview data. Line-by-line coding was discussed by two researchers, and once they faced with divergence in the process of encoding, a special group session of the research team discussed and revised the codes together until agreement could be reached. (3) Similarly, differences in dependent coding were also discussed and refined by the team members until consensus was achieved. (4) According to this coding process, key themes or unified and recurrent ideas which described the participants' opinions and experiences were summed up themes and/or sub-themes. (5) A set of transcripts with coding was categorized and generated into final themes. A memo could be used to record some valuable perspectives, the preliminary ideas and/or ideas transformation in the process of 


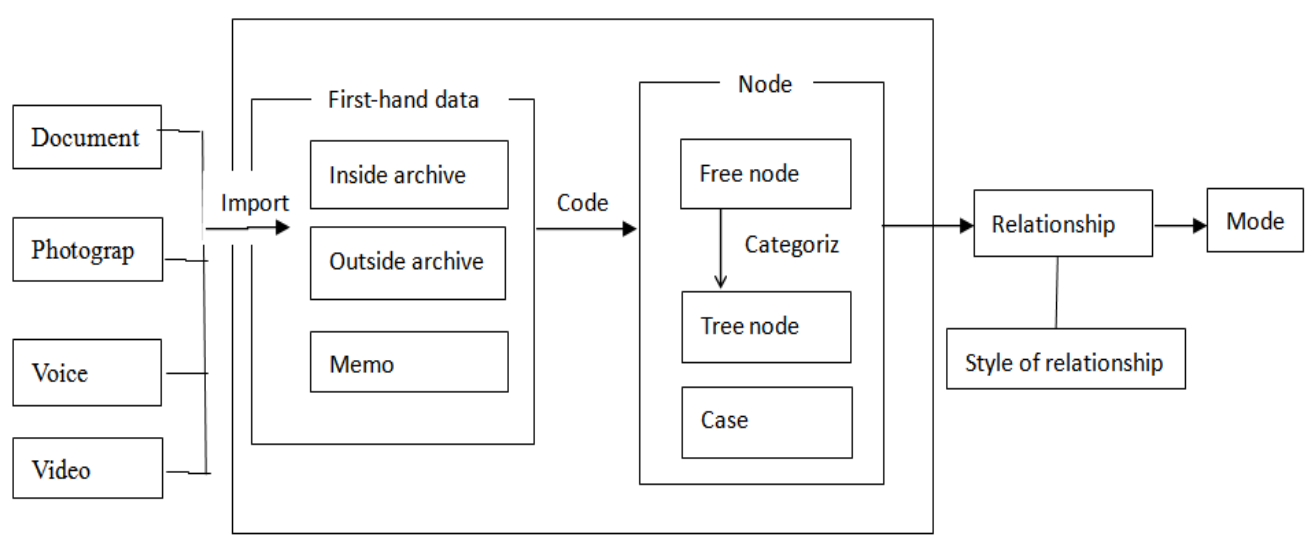

Figure 2: The analysis process of qualitative research.

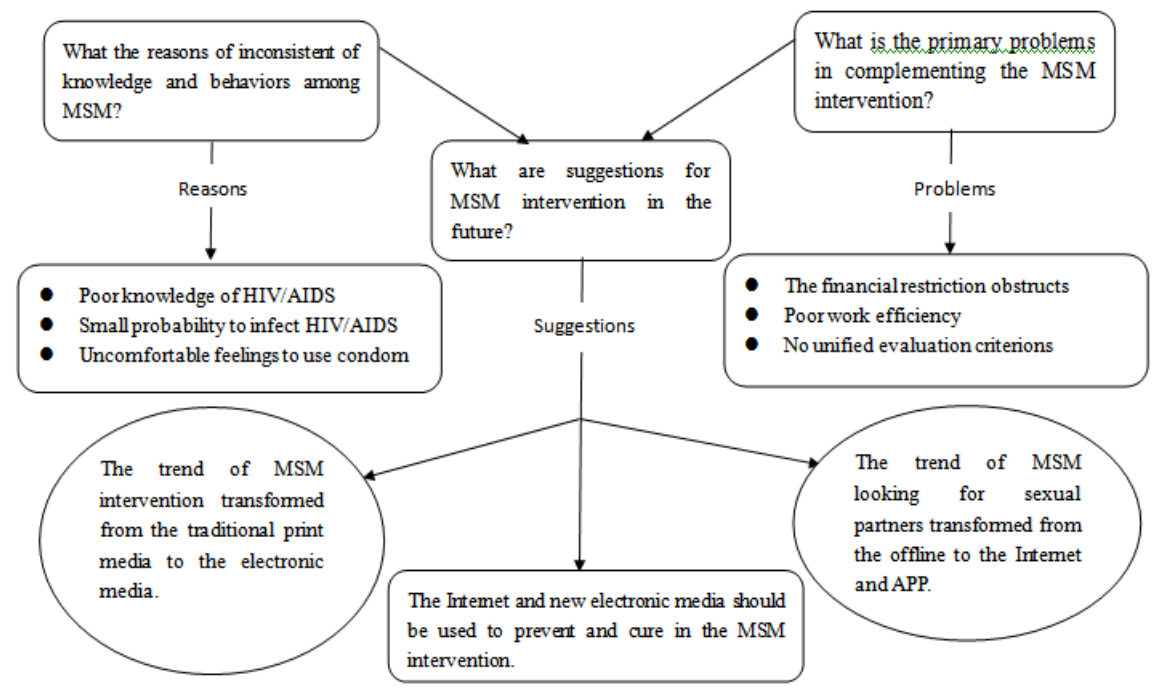

Figure 3: The diagram of key themes.

analysis. (6) Last but not least, the research team evaluated the data and results and verified whether the results were reasonable or not.

\section{Results}

\section{Characteristics of participants}

Of 10 participants interviewed, 5 came from CDCs while 5 came from NGOs, with ages ranging from 25 to 52 (Table 1). Seven of the 10 participants were male and the rest were female. All the participants from CDCs had a bachelor degree or higher education, while only one person from NGO had a bachelor degree. The average working years of participants from the CDCs and NGOs were 15.6 \pm 7.20 and $12.6 \pm 10.95$. All of them worked on MSM intervention for more than 3 years in Hubei province.

\section{Four key themes identified}

Some sub-themes with supported quotes were under four key themes. The following were four key themes concluded based on the interviewers' views and experiences regarding to MSM intervention. And the relationships of key themes showed in the (Figure 3).

1. Poor knowledge of HIVAIDS, small probability to infect
HIV/AIDS and uncomfortable feelings contributed to the inconsistent of knowledge and behaviors among MSM.

When talked about the knowledge and behaviors of HIV/AIDS, most of the interviewers expressed the MSM had poor cognitive and took risk behaviors. And we asked the interviews for nonuse condom as example to explain in both CDCs and NGOs.

General speaking, quite a number of uninfected MSM hold a luck mental of small probability to infect HIV/AIDS. They did not wear condoms because of impossibility to be infected as long as they were careful enough.

\section{------Interviewer F from NGO}

The gay bars, ballrooms, bathrooms and KTVs were the main sites for gathering MSM together, where promiscuous sexual behaviors happened frequently. And it was impossible for these places to forbid someone who had AIDS to enter. Only when MSM improved their knowledge and used condom voluntarily, can keep them from infecting HIV/AIDS. But in fact, the utilization rate of condoms was low. 
Table 1: The characters of respondents for in-depth interviews.

\begin{tabular}{|c|c|c|c|c|c|c|}
\hline \multirow{2}{*}{ No. } & Gender & Age & Level of education & Position & Working years & Administrative region \\
\hline & (male/female) & (year) & (degree) & (grade) & (year) & \\
\hline \multicolumn{7}{|c|}{ CDC group } \\
\hline A & M & 51 & Bachelor & Senior & 25 & Wuhan City \\
\hline B & $\mathrm{F}$ & 43 & Master & Senior & 18 & Wuhan City \\
\hline $\mathrm{C}$ & $\mathrm{F}$ & 47 & Bachelor & Senior & 15 & Wuhan City \\
\hline D & M & 42 & Bachelor & Senior & 15 & Jingzhou City \\
\hline$E$ & $\mathrm{~F}$ & 29 & Master & Intermediate & 5 & Huangshi City \\
\hline \multicolumn{7}{|c|}{ NGO group } \\
\hline $\mathrm{F}$ & M & 26 & High school & Freelance & 3 & Wuhan City \\
\hline G & $M$ & 34 & Bachelor & Teacher & 8 & Wuhan City \\
\hline I & $M$ & 52 & High school & Businessman & 28 & Jingzhou City \\
\hline $\mathrm{J}$ & M & 48 & High school & Businessman & 20 & Huangshi City \\
\hline
\end{tabular}

A number of MSM knew it would increase the risk of HIV/AIDS infection without using condoms, but for the reasons of reducing sexual stimulation, sexual partner's indisposition and promiscuous sexual behavior, the usage of condoms was low.

\section{------ Interviewer G from NGO}

2. The financial restriction obstructs, poor work efficiency and no unified evaluation criterions were the primary problems in MSM intervention.

The MSM intervention was facing with, some intractable problems, such as funds shortage, unreasonable funds allocation, pinch of staff, nonstandard management, difficult to unify evaluation criteria.

In my opinion, the dominating problem we faced were funds in Hubei province. And different levels of CDCs faced with different funding problems. In the CDCs of central city, the funds could not be rational disposition. While in the district level of CDCs, they were lack of funds, which lead to MSM intervention activities could not be implemented. Similarly, in the NGOs, for lack of enabling financial, legal and political environment, few of them obtain registered status. Thus, they are not eligible to apply governmental funds.

\section{------ Interviewer B from CDC}

Another serious problem was poor work efficiency in CDCs and NGOs. Especially in NGOs, no enough specialists, no standardized management and no unify evaluation criteria made low work efficiency.

The people in NGOs were volunteers with the low level of education. Most of them did not receive undergraduate education and knew little about disease prevention, statistical analysis or professional assessment. To finish the work targets, they did HIV/ AIDS testing repeatedly. Besides, with weak supervision of CDCs, the intervention work was inefficiency.

\section{------ Interviewer C from CDC}

There were no unified evaluation criterions for MSM intervention in China. The different intervention organizations conducted MSM interventions and evaluated the effects independently.. In this case, it was difficult to supervise and evaluate the intervention work.

\section{------ Interviewer $\mathrm{H}$ from NGO}

3. The MSM intervention work in CDCs and NEOs should coordinate with each other.

The differences existed in funds, intervention strategies, intervention sites and intervention times between CDC and NGO, they should coordinate with each other and make great efforts to reduce HIV/AIDS infection among MSM.

CDC, as a special institution for HIV/AIDS prevention, was composed of experts in medicine, epidemiology, hygiene and statistics. The work team was so professional and MSM intervention could conducted more efficiently. Especially in the CDCs of central city, available funds and standard management were the two obvious advantages.

\section{------ Interviewer D from CDC}

An apparent disadvantage was staff shortage. In the district level of CDCs, there was not enough staff in the department of AIDS and each one was responsible for different tasks, someone for lab testing, someone for VCT, someone for the commercial sexual workers, someone for MSM, and someone for floating population. Everyone must finish their tasks on time to meet the performance assessment.

\section{------ Interviewer E from CDC}

NGOs provided free AIDS testing, free condoms, free petroleumbased lubricants, and some intervention manuals to MSM timelessly, which can improve the knowledge of AIDS prevention and reduce risk behaviors. However, it was not free forever.

With shortage of staff in CDCs, the government allotted most intervention tasks to NGOs. In general, it was difficult for individual MSM to set up a well-trusted relationship with government bodies. Peer education was the most common measure in MSM intervention, and depending on one MSM to educate and influence other MSM 
was proved to be effective. In terms of peer education intervention, NGOs had more advantages than CDCs, because they were usually at a better position to advocate for changes in health policies, allocation of resources, and rights for their community.

\section{------ Interviewer I from NGO}

NGOs conducted peer education also took negative effects. Frequent peer education expanded the scope of friends and increased the frequency of communication. The more communication, the more risk behaviors.

\section{------ Interviewer J from NGO}

4. The Internet and new electronic media should be used in the MSM intervention.

The Internet and new media had been used for making and keeping touch with friends for many years. But both CDCs and NGOs rarely used the Internet and media for MSM intervention.

With the development of society, Internet and mobile phones were used generally. More and more MSM population made friends by Application (APP) services. With GPS function, the social contacts were not limited in the parks, gay bars, bathrooms and some other traditional public places, especially when some professional gay dating APPs, such as Grindr, Jack' d, Blued and Zank, were developed and applied. If we can make good use of those APPs to send messages of health promotion and health education, it would be timesaving and high-efficiency.

\section{------ Interviewer A from NGO}

Grindr was the largest gay social network APP in the world, which had more than 10 million users in 192 countries now. And over 2-million users daily took active in the APP to Chat, share photos, and meet up with guys nearby. In China, it was Blued that used widely, which owned the largest concurrent users in the same network software.

In the developed countries, medical workers used the Internet or mobile phones to send healthy prevention messages and improve HIV/AIDS medication adherence. Researchers utilized the voice or text messaging functions to conduct phone-based HIV intervention at first; then were the APP and mobile web of smart-phones; until now, it was Blued. The MSM intervention based on smart-phones abroad had been proved effect and we should learn from it.

\section{----- Interviewer G from NGO}

\section{Discussion}

There were two types of intervention organization managed and guided the HIV/AIDS prevention and control in China. And one was called CDC, an official agency specialized for the disease prevention and control; another was named NGO, whose members were from social organizations, enterprises, hospitals, and individuals. CDCs mainly played management, supervision and evaluation role in the MSM intervention, with health education and health promotion regularly. However, in grass-roots of CDCs, there were few MSM intervention activities resulting from lack of money, short of hands, no appropriate service delivery sites and setting. Thus, most tasks were entrusted to NGOs whose members were derived from the local, and they could conduct MSM intervention smoothly and favorably via peer education. The intervention measures including voluntary counselling and testing, sending condoms, sending lubricants and other related health education conducted in NGO irregularly. In addition, with deficient supervision mechanism of CDC, it was difficult to assess the intervention effect in NGO because of no unified evaluation criteria, no periodical supervision and no reasonable guidance. Thus, it is essential to strengthen cooperation on MSM intervention work in two groups.

Qualitative research methods are most utilized in anthropology, the humanities and sociology fields to explore some sensitive problems, such as suicide, homicide, drug abuse, sexual abuse. Existed qualitative researches have little been focused on the MSM intervention [20]. Selecting the staff of MSM intervention in CDCs and NGOs as a target population for interview had several advantages. Firstly, the staff participated in the interview were experts of MSM intervention and worked in CDCs or NGOs more than 3 years. With rich experiences, they had a profound insight on MSM intervention, and their suggestions would offer an important reference for the future intervention work. Secondly, the participants came from different CDCs and NGOs and what they said reflected the level of MSM intervention in different organizations. Combined with the strengths and weaknesses of CDCs and NGOs, they could cooperate with each other. Finally, the interviewers came into contact with MSM by voluntary testing, health education and health promotion, psychological counseling and drug therapy. They were familiar with MSM very well. Through interviewed the specialist, some momentous and covert information which MSM were reluctant to response in the quantitative survey was obtained.

The financial restriction obstructs, poor work efficiency and no unified evaluation criterions were three problems that the intervention organizations faced. For the CDCs, especially the county level of CDCs, because of insufficient funds, they could not conduct intervention activities among MSM. While for the NGOs, it was the inappropriate use of funds that leaded to the poor intervention effect. The NGOs conducted MSM intervention activities by peer education, which took more advantages than the CDCs, but the more peer communication, the more risk behaviors. In addition, the CDCs and NGOs formulated their own intervention mechanisms and evaluated the intervention effects independently. Thus, it was difficult to supervise and evaluate the MSM intervention work.

The knowledge of HIV/AIDS was poor among MSM. Most of the interviewers expressed the MSM had low cognitive about HIV/ AIDS and took risk behaviors. And a review showed that regular male partnerships in Chinese MSM had the lowest consistent condom use (19.9\%) compared with noncommercial casual (30.4\%) and commercial partnerships (58.0\%) during anal intercourse over the past six months [22]. In some commercial entertainment places, including the gar bars, ballrooms and KTV, there was a few MSM called "Money Boys" who were usually younger, less educated, and more likely to have unprotected sex with multiple male clients compared to the broader MSM population [23]. They realized that using condom could protect them from infecting HIV/AIDS clearly, however, for some purposes, these "money boys"did not used condom. Even quite a number of uninfected MSM still hold 
luck mental of small probability to infect HIV/AIDS. They did not wear condoms because they considered that it was impossibility to be infected as long as they were careful enough. Although the MSM received the health education and condom promotion, they still took risk behaviors. The existed intervention measures, ways and skills were lack of effect. Thus, it not only needed to expand the intervention scope and times, but also to pay more attention on the intervention ways and skills to change the risk sexual behavior [16].

With the development of the smart phones and gay dating software, more and more gays making friends by the APP. There are two changing trends of MSM intervention ongoing. One trend of MSM intervention transformed from the traditional print media to the electronic media, especially when the mobile phones appeared and used universally. Some studies used text messaging dynamically to enhance adherence and improve important clinical outcomes for people living with HIV $[1,6]$. Some studies adopted a brief webbased intervention offering cognitive behavioral skills training and motivational enhancement to reduce sexual risk in MSM $[9,12]$. Another trend of MSM looking for sexual partners transformed from the offline to the smart phones and APP. With GPS function, the MSM could chat with anyone who want to talk nearby. And with the APP of Grindr, Jack'd, Blued and Zank, the social contacts were not in the traditional public places but in a chat room. Both the CDC and NGO can utilize the voice, video or text messaging functions to conduct phone-based MSM intervention. In China, Blued and We Chat were main social networking software among MSM, and as new tools of social interaction, they has grown gradually and would become the mainstream of making friends among MSM [24]. If related MSM intervention workers can make good use of those APPs to send messages of health promotion and health education, it would be timesaving and high-efficiency.

In China, no literatures existed in using gay social network APP to conduct intervention among MSM. Considering it had taken important influence on sexual behavior and sexual partners among MSM [18], MSM intervention should focus on sending voluntary counseling testing information, implementing peer education and pushing health knowledge by APPs in the future.

\section{Limitations}

Several limitations existed in this study. Firstly, there was a selection bias. The qualitative research used convenience sampling to recruit interviewers, which may not represent all the staff of MSM intervention. Secondly, the interview results from the study are based on relatively small sample which might decrease the generalizability of the results and limit the heterogeneity of the sampling. Finally, it is inevitable to produce information bias in the qualitative research. The data derived from subjective opinion of the interviewers. However, for some open-ended and sensitive questions, there is no criterion to judge the truth, thus the interview was the best way to explore the sensitive questions. And it is better to combine with the quantitative approaches in future research.

\section{Conclusion}

This study documented the knowledge and suggestions of MSM intervention among staff in CDCs and NGOs. The existed intervention measures in CDCs and NGOs have not taken obvious effect on MSM, and it is necessary to explore new intervention approaches to change risk behavior of sexual. An approach of combining with smart phones and gay social network APP, should be used in MSM intervention in the future.

\section{Acknowledgement}

The authors would like to thank the staff who participated in the study, and thank the Wuhan Centers for Disease Control \& Prevention (CDCs) and Non-government Organizations (NGOs) in Hubei Province for their contribution to AIDS prevention.

\section{Author Contributions}

WZ and XDT designed the experiments. XM and YW performed the experiment. XXL and YW analyzed the data. XXL and XDT contributed to writing the manuscript.

\section{References}

1. Furberg RD, Uhrig JD, Bann CM, Lewis MA, Harris JL, Williams $P$, et al. Technical Implementation of a Multi-Component, Text Message-Based Intervention for Persons Living with HIV. JMIR research protocols. 2012; 1: 17.

2. Rendina HJ, Jimenez RH, Grov C, Ventuneac A, Parsons JT. Patterns of lifetime and recent HIV testing among men who have sex with men in New York City who use Grindr. AIDS and behavior. 2014: 18, 41-49.

3. Higa DH, Crepaz N, Marshall KJ, Kay L, Vosburgh HW, Spikes P, et al. A systematic review to identify challenges of demonstrating efficacy of HIV behavioral interventions for gay, bisexual, and other Men who have Sex with Men (MSM). AIDS and behavior. 2013; 17: 1231-1244.

4. O' Donnell L, Stueve A, Joseph HA, Flores S. Adapting the VOICES HIV behavioral intervention for Latino men who have sex with men. AIDS and behavior. 2014; 18: 767-775.

5. Holloway IW, Rice E, Gibbs J, Winetrobe H, Dunlap S, Rhoades H. Acceptability of smart phone application-based HIV prevention among young men who have sex with men. AIDS and behavior. 2014; 18: 285-296.

6. Reback CJ, Grant DL, Fletcher JB, Branson CM, Shoptaw S, Bowers JR, et al. Text messaging reduces HIV risk behaviors among methamphetamineusing men who have sex with men. AIDS and behavior. 2012; 16: 1993-2002.

7. Cohn AM, Hunter-Reel D, Hagman BT, Mitchell J. Promoting behavior change from alcohol use through mobile technology: the future of ecological momentary assessment. Alcoholism, clinical and experimental research. 2011; 35: 2209-2215

8. Phillips G, Magnus M, Kuo I, Rawls A, Peterson J. Use of Geo Social Networking (GSN) mobile phone applications to find men for sex by Men who have Sex with Men (MSM) in Washington, DC. AIDS and behavior 2014; 18: 1630-1637.

9. Grosskop FNA, Le Vasseur MT, Glaser DB. Use of the Internet and mobilebased "apps" for sex-seeking among men who have sex with men in New York City. American journal of men's health. 2014; 8: 510-520.

10. Young SD, Cumber land WG, Lee SJ, Jaganath D, Szekeres G, Coates T. Social networking technologies as an emerging tool for HIV prevention: a cluster randomized trial. Annals of internal medicine. 2013; 159: 318-324.

11. Muessig KE, Pike EC, Fowler B, LeGrand S, Parsons JT, Bull SS, et al. Putting prevention in their pockets: developing mobile phone-based HIV interventions for black men who have sex with men. AIDS patient care and STDs. 2013; 27: 211-222.

12. Miranda J, Cote J, Godin G, Blais M, Otis J, Gueheneuc YG, et al. An Internet-Based Intervention (Condom-Him) to Increase Condom Use Among HIV-Positive Men Who Have Sex With Men: Protocol for a Randomized Controlled Trial. JMIR research protocols. 2013; 2: 39.

13. Burrell ER, Pines HA, Robbie E, Coleman L, Murphy RD, Hess KL, et al. Use of the location-based social networking application GRINDR as a recruitment 
tool in rectal microbicide development research. AIDS and behavior. 2012 16: $1816-1820$

14. Williams M, Bowen A, Ei S. An evaluation of the experiences of rural MSM who accessed an online HIVIAIDS health promotion intervention. Health promotion practice. $2010 ; 11$ : 474-482

15. Kumar RD, Poluru R, Chandran SA, Alary M, Dandona L, Patterns of condom use by men who have sex with men before and after the Avahan intervention in Andhra Pradesh state of India. BMC Public Health. 2014; 14: 1-8.

16. Chow EP, Lau JT, Zhuang X, Zhang X, Wang Y, Zhang L. HIV prevalence trends, risky behaviours, and governmental and community responses to the epidemic among men who have sex with men in China. BioMed research international. 2014; 607261

17. GUO Wei, WZY, SONG Ai-jun, Pound stone K. Impact of HIV/sexually transmitted infection testing on risky sexual behaviors among men who have sex with men in Lang fang, China. Chinese Medical Journal. 2013; 126: 1257 1263.

18. Qi J, Zhang D, Fu X, Li C, Meng S, Dai M, et al. High risks of HIV transmission for men who have sex with men--a comparison of risk factors of HIV infection among MSM associated with recruitment channels in 15 cities of China. PloS one. 2015; 10: e0121267.

19. Christensen JL, Miller LC, Appleby PR, Corsbie-Massay C, Godoy CG Marsella SC, et al. Reducing shame in a game that predicts HIV risk reduction for young adult MSM: a randomized trial delivered nationally over the Web. Journal of the International AIDS Society. 2013; 16: 18716.

20. Siegler AJ, Voux A, Phaswana-Mafuya N, Bekker LG, Sullivan PS, Baral SD, et al. Elements of Condom-Use Decision Making among South African Men Who Have Sex with Men. Journal of the International Association of Providers of AIDS Care. 2014; 13: 414-423.

21. Maulsby CGM, Lindsey K, Kelley R, Johnson K, Montoya D, et al. A systematic review of HIV interventions for black Men who have Sex with Men (MSM). BMC public health. 2013; 13: 1-13.

22. Chow EP, Wilson DP, Zhang L. Patterns of condom use among men who have sex with men in China: a systematic review and meta-analysis. AIDS and behavior. 2012; 16: 653-663.

23. Chow EP, IU KI, Fu X, Wilson DP, Zhang L. HIV and sexually transmissible infections among money boys in China: a data synthesis and meta-analysis. PloS one. 2012; 7: e48025

24. Landovitz RJ, Tseng $\mathrm{CH}$, Weissman M, Haymer M, Mendenhall B, Rogers $\mathrm{K}$, et al. Epidemiology, sexual risk behavior, and HIV prevention practices of men who have sex with men using GRINDR in Los Angeles, California. Journal of urban health: bulletin of the New York Academy of Medicine. 2013; 90: 729-739.
Austin J Public Health Epidemiol - Volume 4 Issue 2 - 2017 ISSN : 2381-9014 | www.austinpublishinggroup.com Tan et al. (C) All rights are reserved
Citation: Liu X, Wang Y, Zhou W, Mei X and Tan X. The Present and Future Tread of MSM Intervention: A Qualitative Study in Hubei Province, China. Austin J Public Health Epidemiol. 2017; 4(2): 1058. 